\title{
LETTER
}

\section{Response to 'Remarkable prevalence of celiac disease in patients with irritable bowel syndrome plus fibromyalgia in comparison with those with isolated irritable bowel syndrome: a case-finding study' - authors' reply}

\author{
Luis Rodrigo ${ }^{1 *}$, Ignacio Blanco ${ }^{2}$, Julio Bobes ${ }^{3}$ and Frederick J de Serres ${ }^{4}$ \\ See related research by Rodrigo et al., http://arthritis-research.com/content/15/6/R201, and
}

related letter by Sherry, http://arthritis-research.com/content/16/1/402

We greatly appreciate Sherry's comments [1] on our recent article published in Arthritis Research \& Therapy [2], and we agree with him that, although the majority of patients with celiac disease (CD) and fibromyalgia syndrome (FMS) may improve with a gluten-free diet (GFD), this may not be a uniform phenomenon. However, we believe that his observations in children [3] cannot be extrapolated to adults, such as those comprising our target population.

Globally, the seven adult females with screeningdetected $\mathrm{CD}$, also categorized in our series as patients with severe irritable bowel syndrome (IBS) and FMS, significantly improved after 1 year on a GFD, with a decrease of $51 \%$ to $60 \%$ in tender points, Fibromyalgia Impact Questionnaire, Health Assessment Questionnaire, and visual analog scales as well as in the number of drugs prescribed, accompanied by an increase of $48 \%$ to $60 \%$ in Short-Form Health Survey (SF-36) physical and mental scores and a reduction of tissue transglutaminase 2 to normal values [3]. However, we observed that some important items of the SF-36 (including social functioning, role emotional, and mental health) were almost unchanged. Therefore, although in general a significant improvement was seen in all outcome measures with $C D$ treatment, the improvement was not uniform but was much more obvious in some cases than in

\footnotetext{
* Correspondence: Irodrigosaez@gmail.com

${ }^{1}$ Gastroenterology, Central University Hospital of Asturias (HUCA), c/ Celestino Villamil, s.n, 33006 Oviedo, Principality of Asturias, Spain Full list of author information is available at the end of the article
}

others. The improvement was statistically significant but always partial, since the total disappearance of all symptoms was not achieved by any of the patients [4].

Since treatment with a GFD is a reasonable and relatively easy therapeutic measure that can significantly increase the quality of life (though not entirely relieve symptoms) of many patients with both FMS and CD, it seems advisable in our opinion to investigate $\mathrm{CD}$ with serology and genetic markers and, if these turn out to be positive, to perform an upper gastrointestinal endoscopy in conjunction with several duodenal biopsies. This will enable the selection of patients with IBS and FMS who are likely to respond to a GFD.

\section{Abbreviations \\ CD: Celiac disease; FMS: Fibromyalgia syndrome; GFD: Gluten-free diet; IBS: Irritable bowel syndrome; SF-36: Short-Form Health Survey.}

\section{Competing interests}

The authors declare that they have no competing interests.

\section{Author details}

${ }_{1}^{1}$ Gastroenterology, Central University Hospital of Asturias (HUCA), c/ Celestino Villamil, s.n, 33006 Oviedo, Principality of Asturias, Spain. ${ }^{2}$ Biomedical Research Office of the Principality of Asturias - FICYT, c/ Rosal 7 -bis, 33009 Oviedo, Principality of Asturias, Spain. ${ }^{3}$ Medicine Department, Psychiatry Area, University of Oviedo, Juan Clavería 6, 33006 Oviedo, Principality of Asturias, Spain. ${ }^{4}$ National Institute of Environmental Health Sciences, Research Triangle Park, Chapel Hill, NC 27709, USA.

\section{Published: 17 Feb 2014}

\section{References}

1. Sherry DD: Response to 'Remarkable prevalence of celiac disease in patients with irritable bowel syndrome plus fibromyalgia in comparison 
with those with isolated irritable bowel syndrome: a case-finding study'. Arthritis Res Ther 2014, 16:402.

2. Rodrigo L, Blanco I, Bobes J, de Serres FJ: Remarkable prevalence of celiac disease in patients with irritable bowel syndrome plus fibromyalgia in comparison with those with isolated irritable bowel syndrome: a casefinding study. Arthritis Res Ther 2013, 15:R201.

3. Taubman B, Mamula P, Sherry DD: Prevalence of asymptomatic celiac disease in children with fibromyalgia: a pilot study. Pediatr Rheumatol Online J 2011, 9:11

4. Rodrigo L, Blanco I, Bobes J, de Serres FJ: Clinical impact of a gluten-free diet on health-related quality of life in seven fibromyalgia syndrome patients with associated celiac disease. BMC Gastroenterol 2013, 13:157.

10.1186/ar4488

Cite this article as: Rodrigo et al.: Response to 'Remarkable prevalence of celiac disease in patients with irritable bowel syndrome plus fibromyalgia in comparison with those with isolated irritable bowel syndrome: a case-finding study' - authors' reply. Arthritis Research \& Therapy 2014, 16:403 